\title{
Historic Architecture and Aesthetic Reconstruction of Guqin Aesthetics
}

\author{
Boyuan Song ${ }^{1}$ \\ School of humanities, Jiangxi University of Traditional \\ Chinese Medicine Jiangxi Nanchang, 330004, China \\ e-mail:6071620@qq.com
}

\author{
Huajie Sui ${ }^{2, *}$ \\ School of humanities, Jiangxi University of Traditional \\ Chinese Medicine .Jiangxi Nanchang, 330004, China \\ e-mail: 510563704@qq.com
}

\begin{abstract}
Guqin instrument, from many historical documents, have fully demonstrated its implication of cultural significance has gone beyond the instrument itself. Piano music in the tradition of thousands of years has been the development of ancient cultural elites constantly enriched and improved aesthetic construction and conscious, so that not only can Guqin, self-cultivation, but also family, country and the world of social function, its cultural connotation far more than other instruments in general. By origin of Guqin. Near Guqin in to inherit and carry forward the traditional culture, based on the proposed reconstruction of contemporary aesthetic sense. Correctly grasp the contact Guqin shape, sound, cultural and aesthetic thought between, with the development trend of particularity and cultural reform of Guqin in Chinese traditional culture as innate, promote Guqin culture. These intellectuals is not only the creator of Guqin treatises and recorded, and those who practice the art of Guqin performances. Confucius period of band is one of the necessary basic training with disabilities groups, this makes their written work is not very empty Guqin aesthetics, broad, but is closely related to piano playing music and other artistic practice.
\end{abstract}

Keywords-Guqi;n musical; aesthetic; culture; ancient intellectual

\section{INTRODUCTION}

Currently, scholars focus on the Guqin aesthetics research focused on closely related to the art of Guqin aesthetics and philosophy of life and other aspects of the Chinese nation, and for the ancient Guqin musician identity writings written research is somewhat lacking [1, 2]. According to the ancient piano music in the aesthetic development characteristics related to the Guqin played it will be divided into three stages, which include early aesthetic perception of Qin, Han, Wei, Jin North when North Korea, the incubation period including the Sui and Tang, Song and Yuan dynasties, flourishing including two of the Ming and Qing dynasties, and ancient piano playing on the aesthetics classify and analyze the statement, trying to sort out the context of the development of piano music played aesthetics, but aesthetics demonstrate artistic practice should be combined with specific conclusions [3].

Research on the History of Music Aesthetics focus can have two, one is musical aesthetic thought philosopher, esthetician; one is a musician discussed issues of music aesthetics [4-8]. Like the Warring States philosophers Mencius, Chuang Tzu, Mo and other views of the music mostly belong to the first category of aesthetic thought of music. Guqin aesthetics research paper mainly focus on the second category, but there is a big difference with the second category. Because most author Guqin familiar aesthetic theory in artistic practice, they also are knowledgeable writer, philosopher; they are familiar with a variety of classics and philosophy of life, art phenomenon mastery in the art of Guqin aesthetics being $[9,10]$.

Qin theory about playing Guqin aesthetics is still in the development stage is fragmented, mostly in the form of easy to understand the theory in the story of the show, though not the system, but its significance as the remarks of Confucius and his students discuss the Analects of Confucius Like people today give inspiration is deep and intriguing. This paper would like to paragraphs described to illustrate representative Qin discourse on Chinese people playing Guqin aesthetics, their main emphasis on the deep affection of understanding music. This is mainly due to the pre-Qin shape is still in continuous improvement and development stage, the presence or absence of thirteen bits emblem scholars did not agree. So in Guqin playing skills limited circumstances, pay more attention to the pursuit of mood music performances, which also formed the art of Guqin thousands of years of tradition to pursue.

\section{CONSTRUCTION AND DEVELOPMENT OF GUQIN}

Qin aesthetics from such close contact have been playing traditional practice, but also to better explain to us: aesthetic or musical aesthetics and so should be combined with specific artistic practice, this works only aesthetic concrete and vivid, not generalities abstraction, in order to enable the reader to understand even more profound and concrete [11].

Aesthetic or musical aesthetics should be combined with specific artistic practice, so as to make the reader understand the aesthetics of a more profound and concrete. Some philosophers, aestheticians and music esthetician has long recognized the problem, they emphasized philosophical and specific, binding to aesthetics and artistic practice, they should be complementary and mutually dialectical [12]. Some major differences between Western and Chinese philosophy were shown in Tab. 1. 


\begin{tabular}{|c|c|}
\hline WesteRn PHILOSOPHY & CHINESE PHILOSOPHY \\
\hline Knowledge & Intuition \\
\hline - clear, complex knowing & $\begin{array}{l}\text { - suggestive, simplistic } \\
\text { knowing }\end{array}$ \\
\hline - talking about metaphysics & $\begin{array}{l}\text { - not talking about } \\
\text { metaphysics }\end{array}$ \\
\hline - duality of one and many & - indivisible one and many \\
\hline $\begin{array}{c}\text { - rational, scientific, } \\
\text { qualitative, }\end{array}$ & $\begin{array}{l}\text { - social, aesthetic, } \\
\text { qualitative, }\end{array}$ \\
\hline limited & unlimited \\
\hline
\end{tabular}

Guqin, also known as Qin, lyre, is the nation's oldest stringed instrument, the piano initially only five strings, so that banjo. Han period was the heyday of Guqin Music creation, Guqin during this period has also been a significant development, guqin shapes are basically stable, future generations are used for the guqin as oriented. Born about Guqin, ancient legend "Fuxi violin", "Shen Nong violin", "Shun as banjo", though not the whole letter, but from another perspective can explain, indeed a very long history of Guqin.

Guqin complex structure outside melodies record is quite troublesome. Therefore, from generation to Guqin after a long period of time, thanks to mouth and heart melodies, melodies propagation speed is extremely slow. With the arrival of Han period melodies creative heyday, there is growing emphasis on documentation and dissemination melodies, melodies record has slowly been developed, thus creating the original text notation mode spectrum. Later, Sui and Tang dynasties dominate, come one after another Golden Years and Kaiyuan Spirit of art has also been a huge development, a more literal spectrum of more simplified and convenient notation way - by word spectrum emerged. Save spectrum appears after the word, the offspring of notation as a way to use more Guqin notation

mode.
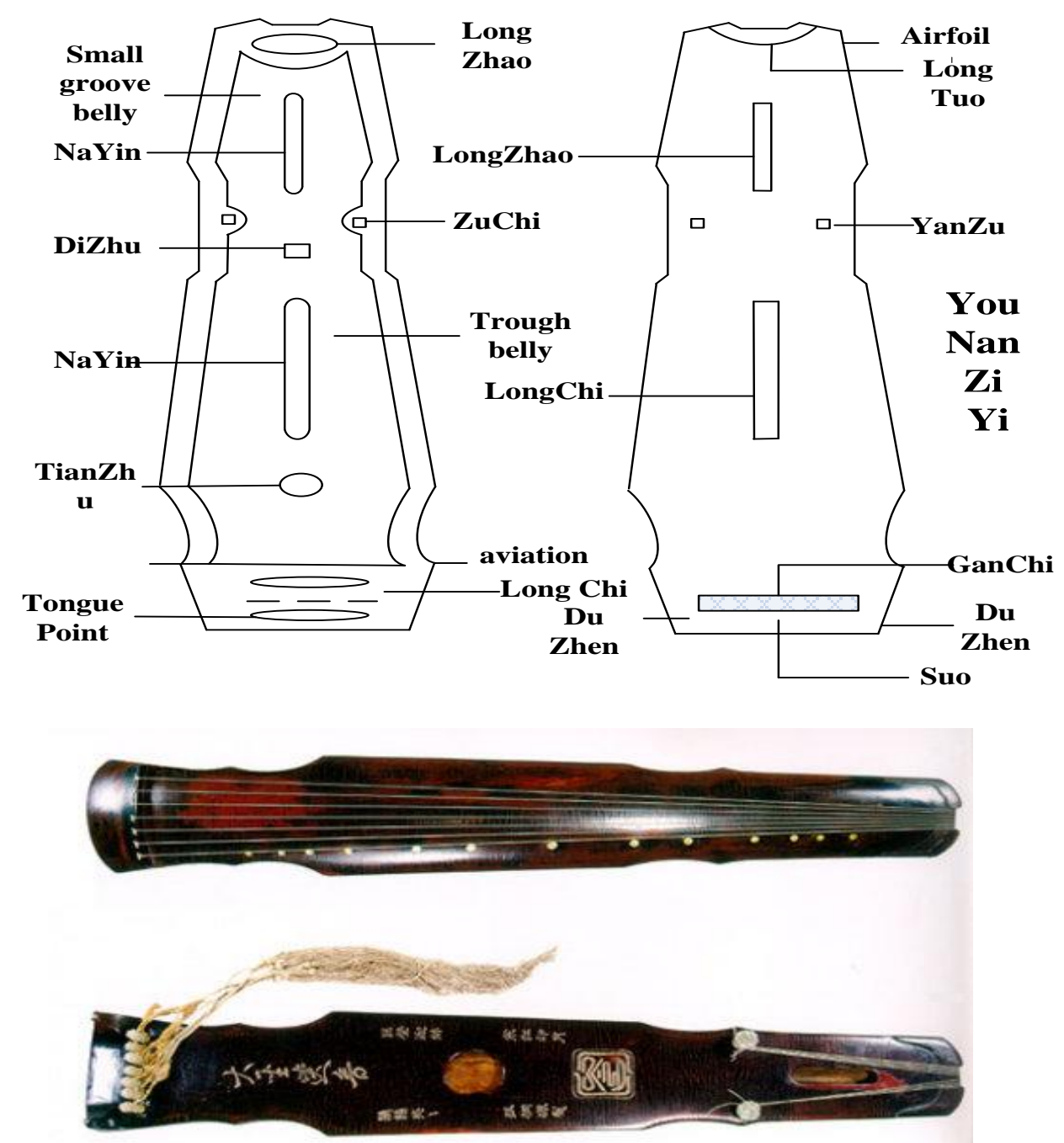

Figure 1. Guqin structure 
Guqin structure was shown in Fig. 1. In addition to often hear guqin seven-string thirteen emblem, as well as the main part of the panel, the floor, the sound beam, frets, pegs, strings and piano legs and the like, complex structure, we want an accurate grasp every Guqin pronunciations, not one or two years of effort is difficult to come down. It is because of Guqin operate fairly trouble, so whether in ancient or in modern times, the guqin is very difficult to popularize.

Guqin notation mode structure and the complexity of the decision and the way of playing the guqin fingering are bound complex. Guqin playing fetch with his left hand Anxian tone, to the right hand playing a chord tone. The main difference Anxian left hand to adjust the tone, Anxian techniques Anxian efforts will be affected. Right hand bomb chord pronunciation Guqin mainly regulated amplitude and intensity. Changes in the larger right-hand playing chords, also do the finger and finger and finger carapace bomb and suicide bombers do not mean face when playing chords. Guqin fingering so complex, requiring violin owned extraordinary perseverance and good finger coordination. This social class people to live and eat as the main pursuit of the ancient peasant economy, the use of so much time and effort to learn these "useless" music, not as much to except from a few grass fields, kill it came a few insects.

\section{HISTORICAL ARCHITECTURE OF GUQIN CULTURAL DEVELOPMENT}

Contending for the academic development can play a strong role in promoting. Guqin cultural nature was well. Guqin culture itself is very personalized, heritage, so it will exist diversified heritage style faction. For example, different regions, different style house modeled after the piano is not the same as each other; the same region, the same piano style home is modeled after its own characteristics and different. So Guqin art, too, and there are many other academic fields as artistic point of view and playing style, so there are many schools, their number, but also most of the traditional musical culture unparalleled. Here, the faction, is in accordance with common moral principles or style and explore different learning groups. Pianist their respective follow some common ideas and style piano track, and a certain pianist groups, the so-called Qinpai. The so-called same Qinpai common characteristics, generally depends on local color, modeled after the origin of the mass spectrum faction is based, views and learn basic piano playing style.

The greatest feature of Chinese Guqin music culture and Chinese philosophical thought, aesthetic thought was the perfect combination, with emphasis on the spiritual qualities of life to enjoy. It absorbed the philosophical thought of Confucianism, Taoism two offshoot. Among the Chinese traditional culture, the qin Confucianism and Taoism culture should be said that embodied in the music synthesizer. Qin Confucian culture mainly by Chiang Kai-shek peaceful, gentle and adapt to the effects of natural Taoism, Great Music sound, clear micro Apprehension and other thoughts. Traditional melodies with five major pronunciation, that is pentatonic, it can be said that the Confucian "and Yazheng" Thought on the implementation of guqin music and piano Yueqing imaginary quiet style and mood of Heaven is mainly Taoism reflection.

Guqin instrument because of its shape, sound, music themes, content, structure and other factors, its musical style is the tendency of static, simple, subtle, ancient pale, feminine, lyrical, elegant beauty. This is the most suitable for the Guqin dead of night, lakeside water play, because such an environment can best fit with the style and mood of their pursuit of Guqin music, to achieve the effect of Heaven. Thus the whole art of Guqin music is called "Qin said," which consist of two words, appreciation and understanding of the guqin is its deep cultural heritage need to dig out the spiritual connotation, the pursuit of "all things and I are one," the spiritual mood, rather than a single piano music tunes from understanding. These primarily reflected in the various aspects of the piano music of the representative of the ideal style and aesthetic standards of Guqin music choice of subject matter, the mood of the pursuit of spiritual and ethical norms.

Guqin culture in development, some of the artists and Qin Qin people are in for Guqin cultural development of the efforts made, they often hold Gathering, concerts and so on, I hope to play the role of propaganda Guqin culture. But Gathering and recitals are mostly played a role in the piano myself and people among themselves, publicity is minimal. Guqin culture rely on the lack of knowledge many times people get the fastest and most complete cultural information media, namely mass communication. Whether radio, television, newspapers, magazines, Internet, have rarely spread to Guqin culture, occasionally appeared in TV shows or movies Guqin sound, but because some of the directors who knew nothing of the guqin and props, coupled with props is zither or Guqin props, the music is also very common in the case of the zither. Due to the lack of these Guqin culture propagation strength, but only in the guqin has many puzzling or cultural misunderstandings, which deeply affected the guqin culture.

With the large number of Western musical culture shifted, many of our musicians accepted and adopted the aesthetic standards of western music, Chinese music will soon locate the stage musical tone, which determines the Chinese music by Western music to guide, all of sound behavior, according to the Western musical culture aesthetic thinking standard to judge. So Chinese music will soon lose its own original national characteristics, giving rise to a "who is the new West, today's modern, who is an old Chinese tradition, yesterday, the ancient" in Chinese music phenomenon, "music" has become Western music terminology. Why Western music from the incoming to today is so fast and widespread impact on Chinese music culture, because, as the main body of Chinese music culture, did not fair and equitable to compare with the Western musical culture, and blindly be incorporated into the Western music culture frame, making the dominant position of Chinese music culture misplaced, or even lost. 


\section{CONCLUSIONS}

Aesthetic theory about the Guqin music piano playing, but Li discussed herein Guqin aesthetics sea of ancient scholars. And in ancient literature author cited only involve playing Guqin aesthetics reflect one side, but even with the new data also probably not the overall grasp and class paper points. Guqin art practice consists of three levels, performance, appreciation and creation, and piano theory, discusses the most detailed information on most aspects undoubtedly played it from the Qin Dynasty to the Ming clear, it has been the focus of attention of many scholars. with the advance of history, its theory from different angles gradually, the Qin Dynasty Guqin shape due to the constraints and other aspects of notation, playing melodies theory is mainly about mood and meaning pursuit, but the aesthetic point of view has been throughout the history of Guqin aesthetics, and is a top priority.

\section{REFERENCES}

[1] Mazur R. Music as a Tool for Self-Realization in Chinese Culture: Based on the Practice of Playing the Guqin[J]. 2015.

[2] WU W, ZHOU X. Originated from the Nature, the Sounds of Guqin Plays for the Breath of Brooks and Mountains: On the Natural Beauty of Chinese Guqin Art[J]. Arts Exploration, 2013, 5: 019 .
[3] Yi-nan L I. Exploration upon the Design Thought of Chinese Guqin: Taking" History of Guqin" as an Example[J]. Hundred Schools in Arts, 2012, 2: 031.

[4] Murong F. The Research on Guqin during the Twenty Years after the "Cultural Revolution"[J]. The New Voice of Yue-Fu (The Academic Periodical of Shenyang Conservatory of Music), 2013, 3: 007.

[5] YU J, YU J. A Study of the Development Countermeasures of Music Performances Aesthetics in China under the Two Historical Important Missions[J]. Journal of Jiangxi Normal University (Philosophy and Social Sciences Edition), 2012, 3: 018.

[6] Boccia L V. Aesthetic Convergences: Comparing Spectacular Key Audibles and Visuals of Athens and Beijing Olympic Opening Ceremonies $[\mathrm{J}]$. The International Journal of the History of Sport, 2012, 29(16): 2264-2275.

[7] Gillan M. " Dancing Fingers": Embodied Lineages in the Performance of Okinawan Classical Music[J]. Ethnomusicology, 2013, 57(3): 367-395.

[8] Christopher K R, He J, Kapur A, et al. Interactive Sound Synthesis Mediated through Computer Networks[C]//Proceedings of Symposium on Sound and Interactivity. 2013: 44-48.

[9] SCHIAVIO A. Commentary on Leman \& Maes, "The role of embodiment in the perception of music"(2014)[J]. Empirical Musicology Review, 2014, 9(3-4).

[10] Beebe N H F. A Complete Bibliography of the Journal on Computing and Cultural Heritage (JOCCH)[J]. 2015.

[11] Shankar S U. Continuous pitch wind musical instrument and a composite string instrument and continuous pitch wind musical instrument: U.S. Patent Application 14/473,251[P]. 2014-8-29.

[12] Wu D, Li C Y, Yao D Z. An ensemble with the Chinese pentatonic scale using electroencephalogram from both hemispheres[J]. Neuroscience bulletin, 2013, 29(5): 581-587. 